\title{
Analysis on the Critical Condition of Active Fault Instability and the Parameters of the Interface Model
}

\author{
Yan-Qiong Liu \\ Institute of Engineering Mechanics, Key Laboratory of Earthquake \\ Engineering and Engineering Vibration, China Earthquake \\ Administration, Beijing 065201,China \\ yanqiong-liu@163.com
}

\section{Ji-Sheng Zhao}

National Earthquake Infrastructure Service, Beijing 100036, China.

\begin{abstract}
In this paper, earthquake triggering is described as the process of local instability of system in mathematical and physical way. And critical instability conditions of the fault in analysis domain are given. Based on elastic-plastic theory general constitutive relationship of the fault plane is derived. And its stability state is discussed by the example of rate and state related friction law corresponding to the characteristic earthquake. The relationship between seismic precursors and fault stability is analyzed in the three conditions of the rock stiffness, the fault interface stiffness and the stress and state of the fault. And the relationship between the parameters of the rate and state related friction law and the repeated interval of characteristic earthquakes is enumerated.
\end{abstract}

Keywords-Earthquake triggering; Critical instability conditions; Interface constitutive relationship; Seismic precursor

\section{INTRODUCTION}

Strain energy is accumulated in underground rock by the long-term tectonic action. When the accumulated energy exceeds a certain limit, underground rock suddenly rupture to form a fault or suddenly slip along existed faults to release the accumulated energy, some of released energy spread out as seismic waves to form ground vibrations, namely, elastic rebound theory, which is the Reed Research 1906 San Francisco earthquake seismogenic fault (San Andreas fault) of surface rupture of the resulting conclusions.

Earthquake can be described as a mechanical problem about the solid internal stability under the condition of the equivalent boundary value. Under the continuous loading of the far field boundary or by the movement of the mantle material, the mediums along the both sides of fault continuously store the strain energy to step deformation of the rock on both sides. On the faults, in the process of strain energy accumulation the obstacles and the concave and convex bodies gradually evolve, resulting in the gradual deterioration of the material and local instability. The strain energy accumulated in the limited area can be suddenly released, which makes the source plane burst rapidly. Elastic rebound produce medium vibration, in the form of wave

\author{
Pei-Xuan Liu \\ National Earthquake Infrastructure Service, Beijing 100036, \\ China. \\ Hong-Suai Liu \\ National Earthquake Infrastructure Service, Beijing 100036, \\ China.
}

propagation, then analysis domain enter a period of adjustment and the new accumulation.

The continuous loading of boundary stress is a long time process. And instability is instantaneous. On the definition of stability, Hill comes down to the follows. If the additional displacement of equilibrium body formed by arbitrary small perturbations keeps infinitesimal the equilibrium state is stable. On the contrary, if any small perturbation can lead to a finite value of the displacement of the object, the equilibrium state is unstable. Obviously, the sufficient condition for the stability of the system is that the internal energy of the object is larger than that of the external force when micro disturbance is applied to the equilibrium position.

In the analysis domain, there is a potential function $\Pi$, which has an increment $\Delta \Pi$ with an arbitrary small disturbance in the equilibrium state. The Taylor series is used to obtain

$$
\Delta \Pi=\delta \Pi+\frac{1}{2 !} \delta^{2} \Pi+\ldots
$$

$\delta \Pi=0$ is sufficient condition for the equilibrium state to be stable. When $\Delta \Pi>0$ and $\delta^{2} \Pi>0$, then the system is stable. When $\delta^{2} \Pi<0$, then the system is unstable. When $\delta^{2} \Pi=0$, then the system is at a critical point. At this time the system may be stable and may not be stable.

In this paper, based on the principle of energy earthquake occurrence is described and converted into local instability problem in mechanics, and the critical condition of fault instability in analysis domain is given. The general constitutive relation of fault plane is derived, and its stability is discussed using rate and state related friction law corresponding to the characteristic earthquake as an example. The relationship between seismic precursor and fault stability is analyzed in three cases of rock mass stiffness, fault interface stiffness and fault stress state. And the relationship between the parameters of the rate and state related friction law and the earthquake recurrence interval is enumerated. 


\section{CRITICAL CONDITION OF FRACTURE INSTABILITY}

The body of the analysis domain is $V$. The physical strength is $b$. The stress boundary is $S_{\sigma}$.The stress is $\phi$.The displacement boundary is $S_{u}$. The displacement is $\bar{u}$.Then the balance equation is

$$
\nabla \sigma+b= \begin{cases}0 & \notin \text { Earthquake } \\ \rho \ddot{u} & \in \text { Earthquake }\end{cases}
$$

Solid constitutive relation is

$$
\sigma=D: \varepsilon \quad \text { or } \quad \dot{\sigma}=D: \dot{\varepsilon}
$$

In the formula, in the linear elastic stage $D=D^{e}$, and In the nonlinear stage $D=D^{e p} \cdot D^{e p}$ is related to the loading condition, the yield condition, the damage condition and the plastic stiffness of the material.

The purpose of this paper is to establish a mechanical model of the earthquake occurrence processes (Figure 1) in domain satisfying the formula (2) (3) under the condition of boundary loading. These include the pseudo-static process of strain energy accumulation and the dynamic process of earthquake triggering.

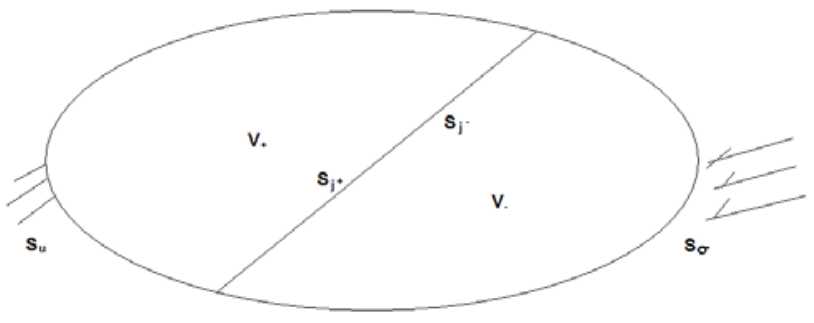

Figure 1. Fracture instability process of solids

There is a displacement discontinuity along the fault in the rock medium, namely, $u^{i}=u+\llbracket u^{i} \rrbracket H_{u}, \quad i=(+,-) \cdot H_{u}$ is step function of displacement discontinuity. Force on both sides of the section is $\phi^{i}$, which depends on $\llbracket u \rrbracket$. The Lagrangian multiplier is introduced to the displacement boundary, then the corresponding potential energy principle is

$$
\begin{aligned}
& \Pi=\int_{V} W d V-\int_{V} b u d V-\int_{S_{\sigma}} \phi u d S-\int_{S_{j^{+}}} \phi^{+} u d S-\int_{S_{j^{+}}} \phi^{+} \llbracket u^{+} \rrbracket d S \\
& -\int_{S_{j^{-}}} \phi^{-} u d S-\int_{S_{j^{-}}} \phi^{-} \llbracket u^{-} \rrbracket d S-\int_{S_{u}} n \sigma(u-\bar{u}) d S
\end{aligned}
$$

In the formula, $W=\int_{0}^{\varepsilon} \sigma d \varepsilon$, and $W$ is strain energy density. $\delta \Pi=0$, then

$$
\begin{aligned}
&-\int_{V} \delta u(\nabla \sigma+b)+\int_{S_{\sigma}} \delta u(n \sigma-\phi)+\int_{S_{j^{+}}} \delta u\left(n^{+} \sigma-\phi^{+}\right)+\int_{S_{j^{+}}} \delta \llbracket u^{+} \rrbracket\left(n^{+} \sigma-\phi^{+}\right) \\
& \quad+\int_{S_{j^{-}}} \delta u\left(n^{-} \sigma-\phi^{-}\right)+\int_{S_{j^{-}}} \delta \llbracket u^{-} \rrbracket\left(n^{-} \sigma-\phi^{-}\right)-\int_{S_{u}} \delta(n \sigma)(u-\bar{u})=0
\end{aligned}
$$

In the formula (5), the first two items on the left are corresponding to the equilibrium equations and the stress boundary conditions. The third and the fifth are the stress compatibility conditions at the two sides of the fracture section, and the seventh is the displacement boundary condition. We are concerned about the fourth and the sixth. Only when the two sides of interface meet the strength conditions, that is $\phi^{+}=n^{+} \sigma_{c}$ and $\phi^{-}=n^{-} \sigma_{c}$, there is the interface slip, namely,

$$
\delta \llbracket u \rrbracket n\left(\sigma-\sigma_{c}\right)=0
$$

By the formula (1), and taking into account that $\sigma_{c}$ is varied in the process of fracture slip, the critical stability condition is

$\delta^{2} \Pi=-\int_{V} \delta \varepsilon(\nabla \delta \sigma) d V+\int_{S_{j^{-}}}\left(n^{-} \delta\left(\sigma-\sigma_{c}\right) \delta \llbracket u^{-} \rrbracket\right) d S+\int_{S_{j^{+}}}\left(n^{+} \delta\left(\sigma-\sigma_{c}\right) \delta \llbracket u^{+} \rrbracket\right) d S=0$

There is a fracture in analysis domain, near which the accumulation of strain energy will be concentrated in the fracture when there is a slip along the interface. And the domain is in from the nonlinear loading stage to the elastic unloading bifurcation stage. At this time the first item on the right of the formula (7) is 0 . Then the criticize condition of fracture instability is

$\int_{S_{j}}\left(n \cdot \delta\left(\sigma-\sigma_{c}\right) \cdot \delta \llbracket u \rrbracket\right) d S=0$

Split the fracture into finite sub segments $S_{j}$, then

$$
\sum_{k} \int_{S_{j k}}\left(n \delta\left(\sigma-\sigma_{c}\right) \cdot \delta \llbracket u \rrbracket\right) d S=0
$$

When the segment without discontinuity is small enough, the formula (9) is equivalent with

$n \delta\left(\sigma-\sigma_{c}\right) \cdot \delta \llbracket u \rrbracket=0$

Based on the formula (3), obtain

$\delta \sigma=D \delta \varepsilon, \quad \delta \sigma_{c}=D_{c} \delta \varepsilon, \quad \delta \varepsilon \propto \llbracket u \rrbracket \otimes n$

The displacement of fault plane is $u=\llbracket u \rrbracket H_{u}$, and $\delta \llbracket u \rrbracket$ is defined as the sliding vector $g$ on the fault plane. Then the formula (10) changes to

$$
\left(n \cdot\left(D-D_{c}\right) \cdot n\right) \cdot g=0
$$

The above formula is the homogeneous linear equation of $g$, and its nonzero solution condition is $\operatorname{det}\left(n \cdot\left(D-D_{c}\right) \cdot n\right)=0 \cdot n$ is the vector in unstable plane direction. When the fracture tendency, inclination and inclination angle are known $n$ is certain. Using coordinate transformation the stress component in the fault plane is 
obtained. Then the critical condition and the unstable condition of the fault plane are

$$
\begin{aligned}
& G-G_{c}=0 \\
& G-G_{c}<0
\end{aligned}
$$

In the formula, $G$ is the shear stiffness of the medium within the fault plane. $E$ is the young's modulus. $v$ is the Poisson's ratio.

To be clear, there is no limit to the stress-strain relation in the above conditions, so these can be elastic matrix and elastic plastic matrix, and also accord with the condition of strain hardening and strain softening.

\section{The Constitutive Relation of Fault Plane}

In mathematics, the fault plane is idealized as a plane with no thickness. In fact, the fault plane has a certain thickness. Its thickness is assumed to be $h$, and then the strain across the fault is

$$
\varepsilon=\frac{1}{h}(\llbracket u \rrbracket \otimes n)
$$

The formula (15) is assumed to be plastic strain. The yield function of the material in the fracture plane width is $\Sigma$. The plastic potential function is $\Xi$.The plastic stiffness coefficient $H$.Then flow rule and plastic consistency condition is

$$
\frac{1}{h}(\llbracket \dot{u} \rrbracket \otimes n)=\lambda \frac{\partial \Xi}{\partial \sigma}, \quad \frac{\partial \Sigma}{\partial \sigma} \dot{\sigma}=H \lambda
$$

Thus, the relation between $\llbracket \dot{u} \rrbracket$ and $\dot{\sigma}$ can be expressed as

$$
\eta\left(\frac{\partial \Sigma}{\partial \sigma}: \dot{\sigma}\right) \frac{\partial \Xi}{\partial \sigma}=(\llbracket \dot{u} \rrbracket \otimes n)
$$

In the formula, $\eta=h / H$, and stress $\dot{\sigma}$ is failure stress $\dot{\sigma}_{c}$.

Based on the formula (17), the explicit relationship (18) between the sliding velocity / distance and the failure stress of the sliding surface can be constructed.

$$
\left(\dot{\sigma}_{c}\right)=F(\llbracket \dot{u} \rrbracket)
$$

Different forms of $F(\circ)$ can be constructed according to different stress failure criteria. Rate and state related friction law is used to describe the periodic stick slip (characteristic earthquake), which some papers have discussed. Rice in 2001 presented the general forms (19) of rate and state related friction law.

$$
\dot{T}=\frac{a \Omega_{0}}{\dot{\delta}_{0}} \ddot{\delta}+(f-\alpha) \dot{\Omega}-\frac{\dot{\delta}_{0}}{L_{c}}\left[T-T_{s s}(\Omega, \dot{\delta})\right]
$$

In the formula(19), $T_{s s}(\Omega, \dot{\delta})=f \Omega_{0}+f\left(\Omega-\Omega_{0}\right)+\frac{(a-b) \Omega_{0}}{\dot{\delta}_{0}}\left(\dot{\delta}-\dot{\delta}_{0}\right)$. The normal stress is $\Omega=n \otimes \sigma$.the shear stress is $T=l \otimes \sigma$. The component of sliding rate in tangential stress direction is $\dot{\delta} . n$ is the fault plane normal. $l$ is the sliding direction. $\Omega_{0}$ and $\dot{\delta}_{0}$ are the of the initial state value of $\Omega$ and $\dot{\delta}$. Without the disturbance of normal stress, the formula (19) is simplified as

$$
\dot{T}+\frac{\dot{\delta}_{0}}{L_{c}} T=\frac{a \Omega_{0}}{\dot{\delta}_{0}} \ddot{\delta}+\frac{\dot{\delta}_{0}}{L_{c}} f \Omega_{0}+\frac{(a-b) \Omega_{0}}{L_{c}}\left(\dot{\delta}-\dot{\delta}_{0}\right)
$$

\section{Stability Analysis of the Rate and State RELATED FRICTION LAW}

The differential form of the dynamic equilibrium equation of a single degree of freedom system is

$$
M \dddot{\delta}+\dot{T}+G \dot{\delta}=G \dot{\delta_{0}}
$$

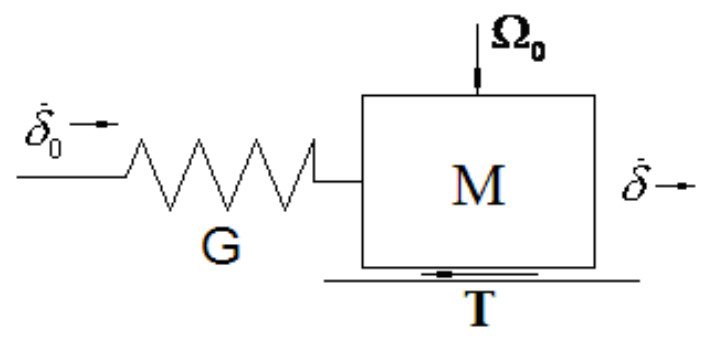

Figure 2. Single degree of freedom model of interfacial friction

Combining (20) and (21) and supposing $\dot{\delta}=A s \exp (s t)+\dot{\delta}_{0}$ and $\ddot{\delta}=A s^{2} \exp (s t) \quad$ and $\dddot{\delta}=A s^{3} \exp (s t)$, the characteristic equation corresponding to the formula (21) is

$$
M s^{3}+\left[\frac{a \Omega_{0}}{\dot{\delta}_{0}}+\frac{\dot{\delta}_{0}}{L_{c}} M\right] s^{2}+\left[\frac{(a-b) \Omega_{0}}{L_{c}}+G\right] s+\frac{\dot{\delta}_{0}}{L_{c}} G=0
$$

When $a>0$ and $b>0$ and $b-a>0$, the critical stability condition is

$$
G-\frac{(b-a) \Omega_{0}}{L_{c}}=0
$$

Obviously, the formula (23) and formula (13) are essentially the same.

\section{FACtORS AfFecting Fault Stability}

\section{A. Basic Characteristics of Active Faults}

The active fault is still active in the nearly tens of thousands of years. Different countries and organizations have a slightly different definition of it, but generally have 4 common principles. (1) The fault has been moving in the present seismic tectonic zone; (2) The fault has the potential to be reactive in the future; (3) There is evidence of recent activity of the fault; (4) The fault is closely related to the seismic activity. A fault should be recognized as an active fault, which must be in accordance with the above 4 principles, and it is best not to violate any one of them. 
Even if a fault is identified as an active fault, the activity scale along the length of the fault is not uniform, and even the character of the sliding is not the same. Therefore, it is an important work to determine section of the basic unit of seismic rupture. It needs to use the earthquake (ancient, historical and modern), the fault (active rate, the activity habit, the geometry shape), the landform shape, the earth physics (exploration, gravity, magnetism), the geological structure background and so on. Various quantitative data on the long-term habit of faults are comprehensively utilized to determine the maximum magnitude of moderate-strong earthquakes occurring in the future.

Further, it is required to determine the parameters of geometry, kinematics and physical mechanics of the fault segment. Geometric parameters include the strike and dip directions, dip angle, length and width of the fault. Kinematic parameters include the rate, direction and property of the fault slip. Physical and mechanical parameters include the strength and stiffness of the fault mud and rock.

\section{B. Characteristic Earthquakes on the Fault Segment}

The rate and state related friction law can be described as a periodic cycle process of stable-slip-recovery, which corresponds to the characteristic earthquake occurrence model of faults. Parameters associated with its stability are represented as the formula (23). These include the shear stiffness of the rock $\mathrm{G}$ and $a, b, L_{c}$ and $\Omega_{0}$ of the fault.

Qualitatively, when $b-a<0$ the system was unconditionally stable and the fault is characterized by state creep. When $b-a>0$ the system was unconditionally stable and the fault is characterized by stick slip.

Suppose $\mathrm{M}$ is $1 \mathrm{Mkg}, \Omega_{0}$ is $50 \mathrm{Mpa}$, $\mathrm{f}$ is 0.6 , $\mathrm{Lc}$ is $10 \mathrm{~mm}$, $\dot{\delta}_{0}$ is $2 \mathrm{~mm} / \mathrm{a}$, a is 0.006 , b is 0.012 , and $\mathrm{G}$ is $25.5 \mathrm{Mpa}$. Solution the formula (20) and (21) to obtained $\delta(t)$ (Figure 3) and the interval of the characteristic earthquake is about 88a. The system parameters of the simulation interval of about 25a are shown in Table 1.

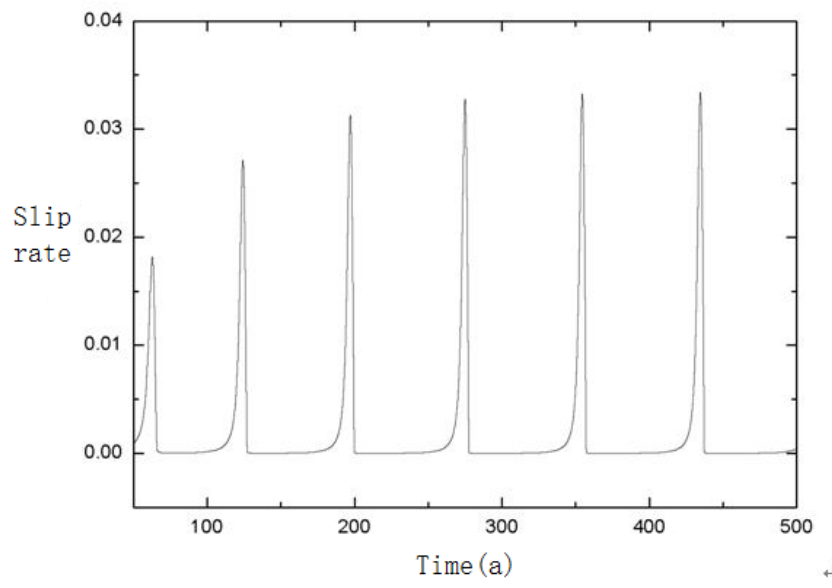

Figure 3. The recurrence interval of characteristic earthquake with single degree of freedom
TABLE I. THE FAULT PARAMETERS OF INTERVAL PERIOD OF ABOUT $25 \mathrm{~A}$

\begin{tabular}{cccccccc}
\hline $\begin{array}{c}\text { sequence } \\
\text { number }\end{array}$ & $\mathrm{G}(\mathrm{Mpa})$ & $\mathrm{a}$ & $(\mathrm{b}-\mathrm{a}) / \mathrm{a}$ & $\begin{array}{c}\dot{\delta}_{0} \\
(\mathrm{~mm} / \mathrm{s})\end{array}$ & $\begin{array}{c}L_{c} \\
(\mathrm{~mm})\end{array}$ & $\begin{array}{c}\Omega_{0} \\
(\mathrm{Mpa})\end{array}$ & $\mathrm{T}(\mathrm{a})$ \\
\hline 1 & 24 & 0.006 & 1.0 & 2 & 80 & 50 & 23 \\
2 & 24 & 0.006 & 0.9 & 2 & 80 & 50 & 24 \\
3 & 24 & 0.006 & 0.8 & 2 & 80 & 50 & 25 \\
4 & 24 & 0.01 & 1.0 & 2 & 80 & 50 & 26 \\
5 & 24 & 0.01 & 1.0 & 2 & 70 & 50 & 20 \\
6 & 22.5 & 0.01 & 1.0 & 2 & 80 & 50 & 27 \\
7 & 24 & 0.01 & 0.8 & 2 & 80 & 50 & 25 \\
8 & 24 & 0.01 & 0.8 & 2 & 80 & 30 & 25 \\
\hline
\end{tabular}

Table 1 example shows that, with the increase of a value (sequence number 1,4), b value decreases (sequence number $1,2,3$ ), the $L_{c}$ value increases (sequence number 4,5 ), $G$ value decreases (sequence number 4,6), and the stability period of the fault increases. With the decrease of the average slip rate $\dot{\delta}_{0}$, the stability period of the fault increases. The change of compressive stress $\Omega_{0}$ has little effect on the stability of the fault (sequence number 7,8), but the peak value of sliding rate increases with the increase of the normal stress.

The above analysis does not take into account the disturbance of the compressive stress $\Omega_{0}$, but it is closely related to the critical condition of fault stability. Static disturbance is related to stress corrosion, fluid pressure change, mining, water storage and drainage of reservoir, High pressure splitting and mining of oil and so on. Dynamic trigger may be the case that far field earthquake wave and earth free oscillation and tidal movement lead to the change of the stress state of different sections in a fault, which causes the failure of the fault.

Although the understanding of some active faults is consistent, there is a certain rhythm of historical and ancient earthquakes in them. Therefore, the critical condition parameters of the local stability of the fault obtained by using the periodic or quasi periodic data of the earthquake have obvious multiple solutions.

\section{Fault Instability Precursor}

There is no difference between large earthquake precursor and fault instability precursor. These include the seismicity anomalies, wave velocity variation of rock, regional crustal deformation, local tectonic stress field changes, abnormal changes of groundwater, geothermal anomaly, gravity anomaly, magnetic anomaly, geomagnetic anomaly and bulge, cracks and subsidence occurred near the fault.

By contrasting the formula (13) and (14), it can be seen that wave velocity variation of rock and geothermal anomaly are directly related to the stiffness $G$. These involve steep rise and steep fall of well water, flower and bubble, temperature fluctuation, changes of spring water flow, hot spring water temperature, oil and gas flow rate, etc. Bulge, cracks and subsidence occurred near the fault and significant deformation cross the fault are related to the fault 
stiffness $G_{c}$, which may be accompanied by an increase or decrease in the number of small earthquakes, resulting in radioactive gas spill. The changes of local tectonic stress field are related to the stress state of fault $\Omega_{0}$, which may involve the disturbance of the small earthquakes in the fault and the large earthquakes in the adjacent area.

The above phenomenon is not uniform distribution of space and time. And it is very important to give a reasonable explanation of the phenomenon related to mechanics. The fault instability may be due to not only the stiffness degradation caused by the plastic stage of the medium on both sides of the fault, but also the stiffness decreases cause by the physical and chemical action of the fault interface medium.

With the GPS layout density and high quality output it is widely used in fault monitoring, for example, paying close attention to the fault section with a high growth rate of local deformation, while identifying the section with a low growth rate, the long-term monitoring of the progress of deformation to sort out the size of the locking region. All these are related to the energy size of the earthquake event caused by fault instability.

\section{CONCLUSION AND SUGGESTION}

Based on the energy principle, the earthquake triggering is converted to the local instability of the system in mathematics and mechanics, and the critical condition of local instability in the analysis domain is given.

Based on the flow rule and plastic consistency condition in the classical elastic-plastic theory, the general form of the constitutive relation of the fault plane is derived. Applying the rate and state related friction law corresponding to the characteristic earthquake its stability is analyzed. And the results are consistent with the critical condition of fault instability.

On the basis of the critical condition of the local instability of the fault, the relationship between the seismic precursor and the fault stability is analyzed, which is based on the three conditions of the stiffness of the rock mass, the interface stiffness and the stress and state of the fault. And the relationship between the parameters of the rate and state related friction law and the repeated interval of characteristic earthquakes is enumerated.

The seismic precursors are divided into three types, which are related to the rock mass and the interface of the fault and the stress state, so that the relationship between the three and the fault instability is clear in the physical and mechanical.

Remarkably, GPS is an important tool, which can not only give the distribution of the displacement field near the fault, but also analyze the energy accumulation and the range of the locked region. Applying them we analyze the location and magnitude of the earthquake that may occur in the future.

\section{ACKNOWLEDGEMENTS}

This work was supported by the National Natural Science Foundation of China under grant No. 51508534, the
National Natural Science Foundation of China under grant No. 51278474, Basic Science and Research Fund for Chinese Commonweal Institutes under grant No.2016A04, Basic Science and Research Fund for Chinese Commonweal Institutes under grant No.2014B06.

\section{REFERENCE}

[1] Xu shifang, li bo. A Dictionary of Seismology.Seismological publishing house, 2000, Beijing.

[2] Stuart W D. Quasi-static earthquake mechanics. Rev. Geophys, Space Phys. ,1979, 17(6):1115-1120.

[3] Ma jin. Uncertainty of the earthquakes and earthquake mechanism and seismic triggering effect of instantaneous factors. Chinese journal of nature.2010,32(6):311-313.

[4] Hill R.On uniqueness and stability in the theory of finite elastic strain.J Mech Phys Solids, 1957,5(4):229-441.

[5] Liu yanqiong, Zhao jisheng. Analysis on Large Earthquakes Sequence of Active Faults near Chuanqing Block Based on GPS.Journalof natural disasters,2014,3(34):58-66.

[6] Washizu K., Variational methods in elasticity and plasticity, Cambridge University Press, 3rd edition, 1982.

[7] K. Ranjith and J. R. Rice, Stability of Quasi-static slip in a single degree freedom elastic system with rate and state dependence friction, Journal of the Mechanics and Physics of Solids, 47, 1207-1218, 1999.

[8] N. Lapusta, J. R. Rice, Y. Ben-Zion and G. Zheng, Elastodynamic analysis for slow tectonic loading with spontaneous rupture episodes on faults with rate- and state-dependent friction, Journal of Geophysical Research, 105, 23,765-23,789, 2000.

[9] J. R. Rice, N. Lapusta and K. Ranjith, Rate and state dependent friction and the stability of sliding between elastically deformable solids, Journal of the Mechanics and Physics of Solids, 49, 1865-1898, 2001.

[10] Changrong He, Tengfong Wong and Nick, Scaling of stress drop with recurrence interval and loading velocity for laboratory-derived fault strength relations, Journal of Geophysical Reseach, Vol. 108, B1, 2037, 2003.

[11] Wu zhonghai,Zhao genmo. The earthquake prediction status and related problems.Geological bulletin of China, 2013,32(10):1493-1512.

[12] Huang gongwen, Wang xiarui, Dang yinqun, Jiang yong. Influence GNSS receiver antenna phase center corrections on precise positioning. GNSS world of China, 2014,39(2):49-53.

[13] Guo baoling. Earthquake recurrence interval and rate and state dependent friction law. Institute of engineering mechanics, China earthquake administration, 2012,Haerbin.

[14] Liu yanqiong. Study on surface permanent displacement of active faults. Institute of engineering mechanics, China earthquake administration, 2013,Haerbin. 